\title{
Ecofriendly Antimicrobial Dyeing for Cotton Fabric Using Natural Extract of Marigold
}

\author{
Dr. Suneeta ${ }^{a}$, Dr. Sujata Harlapur ${ }^{b}$, and Dr. Shantabasavareddi Harlapurc \\ aDepartment of Electronics and Communication Engineering, Vemana Institute of Technology, Bangalore, Karnataka, India \\ ${ }^{b}$ Department of Chemistry, Vemana Institute of Technology, Bangalore, Karnataka, India \\ ${ }^{\mathrm{c}}$ Department of Textile, BVVs S R Vastrad Rural Polytechnic, Guledagudda, Karnataka, India
}

Article History: Received: 11 January 2021; Accepted: 27 February 2021; Published online: 5 April 2021

\begin{abstract}
The main drawback with microorganism's development on fabrics is associated with the maintenance of hygiene and fabric deterioration. In this present investigation a trial has been made to produce an attractive and vibrant textile with ecofriendly antimicrobial dyeing by marigold, a natural resource. Tagetes erecta universally recognized as Marigold is a remarkable source of carotenoids and lutein, grown as a lawn plant. Now a day, lutein is exploding into an indubitably predominant active fitting, employed as an element of the Drugs, Nutrients and Textile Industry. The prospective application of marigold as a characteristic of antimicrobial activity has not been accustomed fully. It is due to the lack of knowledge about its ability and resemblance in material manner. In this work, we have concentrated on the analysis, which was led to deliberate the use of distillate isolate of marigold as an antimicrobial. The antimicrobial competence of the extract was evaluated by coloring on cotton textiles. Testing of the dye ability, its wash quickness and light fastness was done. Educations have validated that antimicrobial coatings have not affected by laundering and shown enhanced colouring effect comprising of fastness to washing, perspiration and rubbing than conservatively dyed textiles. It revealed that the essence of Marigold flower is habitually employed for cotton fabrics as an antimicrobial finish. The samples show very encouraging ends up in terms of antimicrobial resistivity.
\end{abstract}

Keywords: Dyeing; Marigold; antimicrobial dyeing; Fabric.

\section{Introduction}

Microbes are very small organisms, which needs a specific medium to grow, like moisture, temperature, dirt and respective surfaces. Natural fibres are more prone to microbial attack than synthetic fibres; protein fibres act as a nutrient source for moth worms, Cellulosic fibres themselves are not an instantaneous source of nutrients but under appropriate conditions, some fungi secrete enzymes that convert cellulose into glucose - a nutrient source for microorganisms. Also soil, dust and certain textile textures act as a source of supplements for microbes (.Battula, B., 2020; Patil1 DB, 2016).

Microorganism attacks on fibre only when the fibre is damp. Synthetic fibres strongly repel any attack by microorganism due to their high aquaphobicity. These materials and their combinations possess a superior degree of perspiration wetness than textiles, which contain only cotton fibre. In such circumstances there is a more chance of microbial growth on the skin, which results in irritation and smell problems (Roshan S Pai, 2006).

The problems related to microorganism's growth in textiles are with respect to hygiene and fabric deterioration. Microorganism metabolism nutrients, like sweat and soil present in textile products, producing an odour causing intermediate that cause irritation. It results in damage of fabric by causing stain and discolouration or by deteriorating the material itself. Some microbes destroy textures that are present on textiles and a few fungi can eat cotton. Therefore, there is a requirement to inhibit microbiological growth on fabric both in industrial and apparel use.

Today, within the era of eco-friendly operation, it became vital usage to provide hygiene and freshness. But the main constraint is the growth of microorganisms, which are responsible for the decay, staining and odour of textiles. Other than this effect, even microbes can affect human being by spreading diseases and infections. So that it became more significant to protect all apparels, where the prospect of bacterial growth is more and therefore the protection is utmost. This might include the use of medical garments, sanitary napkins, socks, underwear, disposable wipes, carpets etc (Loganathan, J, 2016; Idris, Mohamed, 2016; Vasanth, V., 2017; Teli M. D. MD 2017). In this paper a trial has been made to provide, attractive and vibrant textiles with antimicrobial property.

Nature has gifted many antimicrobial property carrying floras. One such kind is Tagetes erecta universally recognized as Marigold. Here an exploration was done to deliberate the role of the concentrate obtained from marigold petals as an antimicrobial dyeing and considered its dyeing consequences on cotton fabrics. Different mordents were employed to find their influence on colouring.

\section{Material and Method \\ 2.1 Source}


Good quality marigold flowers and leaves were purchased from the former. The dyeing of the cotton fabric was passed through three phases; extraction of dyeing elements from the plant sources, antimicrobial dyeing and mordanting.

\subsection{Fabric}

Plane woven cotton fabric available in the local marketplace was bought for this investigation.

\subsection{Extraction of antimicrobial dyeing element}

The good quality flowers of marigold were cleaned followed by drying under shadow. These dried petals were crushed and boiled with water for 2 hours at $50^{\circ} \mathrm{C}-95^{\circ} \mathrm{C}$. The ratio of fabric and liquid was sustained as 1:50 throughout the extraction process. Extraction was disbursed to urge maximum antimicrobial extract within the result. The extract was then sieved to get clear solution.

\subsection{Scouring of cotton followed by dyeing}

The natural colour dyeing procedure is followed to convey the antimicrobial finish to a material. The bath was prepared by using $3 \%$ of $\mathrm{NaOH}, 2 \%$ of soda and $1 \%$ surface-active agent to clean the cotton fabric. The cotton fabric was dipped in the bath and boiled for 2-3 hours for scouring process and material to liquor ratio has maintained as 1:50 throughout the process (Patil1 DB, 2016; A, Koziróg, 2015). After scouring the fabric was soaked in clean water for half-hour before dying or mordanting. This fabric was coloured with dye extract by maintaining M:L ratio as 1:40. Standard dyeing method has employed for the same and the dyed samples were undergone mordanting.

\subsection{Mordanting}

A mordant is an organic reagent, which supports to form a complex among dye and fabric. In textiles, mordents were used to fix the colour in dyeing exclusively for cotton fabrics. Copper sulphate and alum were used to treat the dyed textile. The mordant solutions with $2 \%$ and $4 \%$ concentrations were prepared depending on the weight of the material. The fabric to liquor ratio was maintained as 1:50. The dyed fabric samples were soaked into two separate mordant baths initially at $60^{\circ} \mathrm{C}$ for half-hour with material to liquor ratio of 1:50. Mordant samples were then dried under shadow.

\section{Evaluation process of sample}

\subsection{Colour fastness tests}

Colour fastness tests signify the standard of the apparel colour. Every fabric after dyeing undergoes colour fastness tests with respect to washing, rubbing, perspiration and light fastness.

\subsubsection{Colour fastness to washing}

It is a standard factor that users wash the material many time within the lifetime of cloth. The variation in colour of the fabric or staining on another garment during washing is usually creates a negative impact on end user. So that for dyed materials the valuation of colour fastness to wash is highly substantial.

\subsubsection{Colour fastness to light}

Sometimes dyed fabrics exposed to sun light they undergo fading or discoloration. So that determination of light fastness test is very important. This test results shows the point at which a dye resists diminishing during sunlit contact. Various colours have various degrees of protection from blurring by light. Generally dyes have certain susceptibility to destruction caused by light. We have to thank these mysterious colours which absorb different wavelengths of sunshine and did not reflect back. Hence resistance of material dyes and prints to degradation largely depends on sun light. Therefore, it is an essential requirement for garments to have high résistance to light fastness. Without this the garments we're not be acknowledged by the customer due to colour change or existence of such colours.

\subsubsection{Colour fastness to perspiration}

Colour fastness towards perspiration is employed to identify the change in colour effect with respect to different types of clothes. The said forms of perspiration may be due to the presence of large quantities of acidic or alkaline salts and which affect the human being.

\subsubsection{Colour fastness to rubbing}

Fastness to rubbing is used to confirm that fabrics will not lose their colour when rubbed with a layer of other cloth / on different materials. This process is also called as crocking and determined with the help of crock meter. It is applicable to dry/wet form of cotton fabrics. In this test cotton fabric (either wet or dry) is rubbed with dyed fabric surface to remove the particles of unfixed dye.

\subsection{Assessment of antibacterial activity}

Qualitative method AATCC 147 and quantitatively by AATCC 100 are used to determine the antibacterial activity of the specimens (Sudha, K,, 2017; Sood, Kaushal,2014; Lall, Winee Surabhi, 2013)

\subsubsection{Qualitative assessment of antibacterial activity}

Antibacterial activity of the sample was tested by adopting the AATCC 147-2011 Parallel Streak Method. The dyed sample and untreated controls of the same is placed in adjacent with previously streaked agar surface by inoculums of a test bacterium. After development, the lively reason of interfered with development underneath and sides of test material specified the antibacterial action of the sample. A standard strain of microscopic 
organisms utilised, which is explicit to the necessities of the material under test. The normal width of a zone of inhibition and a streak on the two sides of the test sample was determined.

\subsubsection{Quantitative analysis of antibacterial test}

Antibacterial test was done using AATCC standard test method 100 for the quantitative assessment of the antibacterial efficiency against staphylococcus aureus bacteria and Klebsiella pneumonia (Panda, 2012; Fatemeh SG, 2012; Lall, Winee Surabhi, 2013). The percentage reduction in bacteria count was calculated by using resulting formulary.

$$
R_{b}(\%)=\frac{(Y-X)}{Y} X 100
$$

$\mathrm{R}_{\mathrm{b}}$ - The percentage reduction in bacteria count

$\mathrm{X}$ - The sum of bacteria's recovered from the inoculated treated test

(Sample strips in the jar incubated with respect to contact period)

Y - The sum of bacteria's of the untreated fabric.

\subsubsection{Wash durability test}

The antibacterial activity of the finished samples was evaluated after being subjected to several wash cycles using AATCC test method 61. The washing was done at MLR of 1:10 with $0.5 \%$ on weight of fabric of AATCC detergent and 100 steel balls at a temperature of $90^{\circ} \mathrm{C}$ for 30 minutes. The cycle of washing was followed by hot rinsing in plain water at $40^{\circ} \mathrm{C}$ for 10 minutes. Later the washed material was tumble-dried. Since a single washdry cycle simulated 8 regular wash cycles, the laundering procedure was repeated for 5, 10, 20 and 30 washes.

\section{Results and discussion}

The colour fastness to washing, light, rubbing and perspiration on dyed cotton sample with alum as a mordant is presented in Table 1.

Table 1: Mordant samples

\begin{tabular}{|c|c|c|c|c|c|c|c|c|}
\hline \multirow[t]{2}{*}{ S1. No } & \multirow[t]{2}{*}{ Mordant } & \multirow{2}{*}{$\begin{array}{c}\text { Mordant } \\
\%\end{array}$} & \multirow{2}{*}{$\begin{array}{c}\text { Colour } \\
\text { Fastness } \\
\text { to } \\
\text { Washing } \\
\text { Grade }\end{array}$} & \multirow{2}{*}{$\begin{array}{l}\text { Light } \\
\text { Fastness } \\
\text { Grade }\end{array}$} & \multicolumn{2}{|c|}{$\begin{array}{c}\text { Colour Fastness to } \\
\text { Perspiration } \\
\text { Grade }\end{array}$} & \multicolumn{2}{|c|}{$\begin{array}{c}\text { Colour Fastness to } \\
\text { Rubbing } \\
\text { Grade }\end{array}$} \\
\hline & & & & & Acid & Alkal ine & Dry & Wet \\
\hline \multirow{2}{*}{1} & \multirow{2}{*}{ Alum } & 2 & 4 & 4 & 4 & 4 & $4-5$ & 4 \\
\hline & & 4 & 4 & 4 & 4 & 4 & $4-5$ & 4 \\
\hline 2 & $\begin{array}{l}\text { Copper } \\
\text { Sulphate }\end{array}$ & 4 & $\begin{array}{c}4 \\
4-5\end{array}$ & 4 & $4-5$ & 4 & $4-5$ & 4 \\
\hline
\end{tabular}

The dyed samples shown good wash fastness of 4 for both mordants alum and copper sulphate at 2 percent concentration. There is no such change in washing fastness with increase in percentage of mordants. But the fabric mordant with copper sulphate shows good to excellent wash fastness of 4-5.

It shows that wash fastness of dye is predisposed by the amount of diffusion of dye and state of dye inside the fibre. The extracted dye from marigold reveals good to excellent wash fastness. It shows the marigold dye have a inclination to aggregate intimate the fiber (thereby growing the molecular dimension) and hence exhibit good wash fastness. The marigold dye easily forms a complex with mordant also has the consequence of in solubilising the dye and creating it colour fast.

The expected performance of dyes and pigments was their fastness to numerous representatives. Sustainability of the dye/ pigment to sunlight is of highest significance. The dyed samples also subject to light fastness test. The samples mordant with $2 \%$ and $4 \%$ concentration of alum and copper sulphate shows good fastness towards light.

The good to excellent light fastness properties of the fabric from Table 1 indicates that close by are no such superficial unsettled natural dyes left over on the fibre apparent after soaping and washing. It anticipated that the natural dye was lanced well inside the fibre abysses. Probably natural dye might have been fixed well by coordinated complex formation with the mordants and mordanting assistant, and also by hydrogen bonding with 
the help of $\mathrm{OH}$ groups of the fibre. The greater laundry property of the colorant was due to the kinetics and thermodynamic things of the metal complex formation and alkaline media used in dyeing..

The perspiration fastness values for $2 \%$ of alum and $2 \%$ of copper sulphate mordants shown good fastness in presence of alkaline medium. The fastness values remain constant as 4 even with increase in percentage of mordants in presence of alkaline medium. It indicated that there is no effect of high percentage of mordents in alkaline medium. Whereas in presence of acidic medium, the sample mordant with $4 \%$ copper sulphate showed good to excellent perspiration fastness. Other than that the fastness values are good for $2 \%$ and $4 \%$ of alum and $2 \%$ of copper sulphate.

The perspiration test data shows with visible variations in the dyed samples after handling under the perspiration solutions. It is due to aspects like the ionization of some of the dye particles throughout an alkaline perspiration test / detachment of dye particles from the substrate of the dye-fiber bond.

Colour alteration to dry rubbing for the canned example is found with superb resistance in the series of 4-5. It is observed that during dry rubbing there is no colour discoloration ranged between no staining and negligible staining (4 to 5). The section shows that very good rubbing fastness even for wet rubbing. Moderate to good rub fastness properties in all these cases indicates that there were almost no superficial unfixed natural dyes left on the fibre apparent after soaping and washing. This natural dye is penetrated well intimate the fibre voids due to the formation of the complex with the mordant and mordanting assistant. Dyed fabric with alum gives always lighter shade. Whereas dyed fabrics with copper sulphate gives always darker shade.

The antibacterial assets of resources were calculated by qualitative (AATCC 147) as well as quantitative (AATCC 100) test methods. The qualitative test is noble for analysing the core mediator/dyed fabrics provided the anti-bacterial mediator used are capable of leaching out. Quantitative test is best sign of amount of antibacterial activity when the bacterial mediators are fixed on to textile material or are unable to leach out. Based on this consideration different tests are carried.

Antibacterial activity of marigold flower extract dyed fabric, mordanted with alum and copper sulphate with qualitative and quantitative methods are given in table 2 and table 3 respectively.

Table 2. Antibacterial activity of marigold flower extract dyed fabric using qualitative method.

\begin{tabular}{|r|r|c|c|c|}
\hline \multirow{2}{*}{ S1 No. } & \multirow{2}{*}{ Particulars } & \multirow{2}{*}{ Mordant } & \multicolumn{2}{|c|}{ Zone of Inhibition in mm } \\
\cline { 3 - 5 } & & & Staphylococcus aureus & Klebsiella pneumonia \\
\hline 1 & Untreated Fabric & - & 0 & 0 \\
\hline 2 & Dyed fabric & Alum & 9 & 6 \\
\hline 3 & Dyed Fabric & Copper Sulphate & 11 & 8 \\
\hline
\end{tabular}

Parallel streak method results clearly shown that marigold dyed fabric having better anti-bacterial properties to both gram positive and negative organisms. The cured cloths do not permit the progress of bacteria under test sample. Dyed samples show zone of inhabitation in the range of 9-11 $\mathrm{mm}$ for Staphylococcus aureus and 6-8 $\mathrm{mm}$ for Klebsiella pneumonia.

Table 3: Antibacterial activity of marigold flower extract dyed fabric using quantitative method

\begin{tabular}{|c|c|c|}
\hline \multirow{2}{*}{ Fabric } & \multicolumn{2}{|c|}{ Bacterial Reduction Percentage } \\
\cline { 2 - 3 } & Sylococcus aureus & K. Pneumoniae \\
\hline Untreated (Control) & 0 & 0 \\
\hline Mordant Alum & 99.96 & 99.76 \\
\hline Mordant Copper Sulphate & 99.97 & 99.94 \\
\hline
\end{tabular}

The percentage reduction value of both the microorganism found to be more than 99 percent for dyed fabrics. Whereas the untreated fabric shows zero percent drop in both microorganisms. The region of reticence standards indicated that marigold flower extract will not preclude the development of bacteria on the cloth, leach out and kills the bacteria. This is an arrangement with the assessment outcomes of percentage drop of standards as shown in table 3 . When a mordant is used the dye forms a complexes with mordant, which is unsolvable and hence it cannot leach out to give a region. The dye now works as an active antimicrobial mediator of the bound type. 
The plant based antimicrobial agents have enormous therapeutic potential as they do not impose any side effects to the human beings. Further, it was observed that numerous plant extract might stay active in contrast to both Gram positive \& negative bacteria contingent on the category of mechanisms existing in the extract.

Antibacterial activities of a textile must be durable to repeated home laundering. This benefit has to be realized for the life span of the fabric. Hence the wash durability test of marigold flower extract dyed fabric was carried out using quantitative method and the results were presented in table 4.

Table 4 Wash durability test results of Marigold flower extract dyed fabrics by quantitative method.

\begin{tabular}{|r|c|c|c|c|}
\hline \multirow{2}{*}{$\begin{array}{c}\text { No of } \\
\text { wash }\end{array}$} & \multicolumn{3}{|c|}{ Mordant } \\
\cline { 2 - 5 } & Sylococcus aureus & K. pneumoniae & Sylococcus aureus & K. Pneumoniae \\
\cline { 2 - 5 } & 99.96 & 99.76 & 99.97 & 99.94 \\
\hline 5 & 92.80 & 92.70 & 93.85 & 93.65 \\
\hline 10 & 86.30 & 85.80 & 88.40 & 87.80 \\
\hline 20 & 81.2 & 80.60 & 83.65 & 82.75 \\
\hline 30 & 73.20 & 71.80 & 75.60 & 74.80 \\
\hline
\end{tabular}

It has been found that virtuous confrontation to bacterial attack. Same time such treated cloths showing gradual decrease in bacterial activity to washes in each cycle. The dyed cloth is tested after many washes. The effect of dyeing remained same. Be that as it may, the antibacterial properties declined essentially subsequent to washing, especially on introduction to light. It is a known factor that from past examinations that iron and some other metallic mixes are fundamental components for the development of microorganisms. The conceivable purpose behind this decrease is that washing and presentation to light would cause some deterioration and subsequently, more iron would be accessible to the microorganisms.

\section{Conclusion}

Common colour can be effectively extricated from blossoms of marigold. Entire cycle of abstraction is ecoaccommodating. With extraction process the antimicrobials are extracted. The coloured samples shows great fastness towards light, wash and sweat. Fabric with medium to excellent fastness properties indicated no such unpredictable natural dye port on cloth apparent even later shampooing and clothes wash. It indicates the natural dye was speared fit inside the fabric abysses and possibly fixed well.

The colour fastness test authenticated the procedures followed for dyeing especially with the natural resources are precise. It is found that very good resistance to bacterial attack by the fabric dyed with marigold extract. At the same time such treated fabrics showing gradual decrease in bacterial activity to washes in each cycle. They got outcomes have demonstrated the colouring capability of marigold blossoms as a phenomenal hotspot for material colouring against microbial. The waste is biodegradable and can be utilized as excellent manure.

\section{Acknowedgement}

Authors are grateful to Vemana Institute of Technology for providing basic laboratory facility. Also wish to thank management of BVVs S.R.V. Polytechnic, Guledagudda for continuous support.

\section{References}

1. A, Koziróg, Wysocka-Robak, A., \& Przybysz, K. (2015). Antifungal activity of paper modified with ionic liquids. Fibres \& Textiles in Eastern Europe

2. Battula, B., Anusha, V., Praveen, N., Shankar, G., \& Latchoumi, T. P. (2020). Prediction of Vehicle Safety System Using Internet of Things. Journal of Green Engineering, 10(4), 1786-1798.

3. Fatemeh SG, Ali SN, Sayed MM, Daryoush A, JavadM.(2012) The effect of mordant salts on antibacterial activity of wool fabric dyed with pomegranate and walnut shell extracts, Coloration Technology.

4. Garikapati, P., Balamurugan, K., Latchoumi, T. P., \& Malkapuram, R. (2020). A Cluster-Profile Comparative Study on Machining AlSi $7 / 63 \%$ of SiC Hybrid Composite Using Agglomerative Hierarchical Clustering and K-Means. Silicon, 1-12.

5. Idris, Mohamed, et al. Effect of temperature on partition behaviour and thermodynamic characteristics of 
bioactive phenolics transfer in aqueous two-phase extraction system and evaluation of antimicrobial activity. International Journal of Chemical \& Petrochemical Technology 5: 1-8.

6. Latchoumi, T. P., \& Parthiban, L. (2018). Advanced Overlap Community Detection by Associative Rule Mining and Multi-View Ant Clustering. International Journal of Engineering and Technology, 7, 21-29.

7. Lall, Winee Surabhi, Amit Alexander Charan, and Akhilesh Bind. (2013). Antimicrobial activity of methanolic and acetonic extracts of Azadirachta indica, Saraca asoca and Curcuma longa. International Journal of Medicine and Pharmaceutical Sciences 3.2 79-86.

8. Loganathan, J., Janakiraman, S., Latchoumi, T. P., \& Shanthoshini, B. (2017). Dynamic Virtual Server For Optimized Web Service Interaction. International Journal of Pure and Applied Mathematics, 117(19), 371-377.

9. Loganathan, J., Latchoumi, T. P., Janakiraman, S., \& parthiban, L. (2016, August). A novel multi-criteria channel decision in co-operative cognitive radio network using E-TOPSIS. In Proceedings of the International Conference on Informatics and Analytics (pp. 1-6).

10. Patill DB, Patil KN, Gaikwad PV, Patil PJ, Shewale UL, BhamburdekarSB.(2016) Extraction of natural dye from rose flower for dyeing cotton fabrics.Int. J. Innovative Research in Multidisciplinary field, $p$ 133.

11. Panda, Sujogya Kumar. (2012): Screening methods in the study of antimicrobial properties of medicinal plants.International Journal of Biotechnology and Research 2.1 1-35.

12. Roshan S Pai, Rajan V. R and Roshan Paul.( July/Sep-2004) Synthetic Fibres, 31(29).

13. Sathyanarayanan, MP, Chaudhari MP, Bhat NV(2011) Development of durable antibacterial agent from ban-ajwain seed (Thymus serpyllum) for cotton fabric, IJFTR, $p$-234.

14. Sudha, K., (2017). et al. "Determination Of Antimicrobial Activity Of Fruits." Int J Med Pharm Sci 7(18).

15. Sood, Kaushal, and R. N. S. Yadav. (2014). Effect Of Solvent On The Extraction Of Antioxidant And Antimicrobial Components From Eryngium Foetidum L. International Journal of Medicine and Pharmaceutical Sciences 4(31-40).

16. Teli M. D. MD, Javed S, MarutiK.(2013) Ecofriendly dyeing and antibacterial finishing of soyabean protein fabric using waste flowers from temples. Textiles and Light Industrial Science and Technology (TLIST). $p$ - 78.

17. Vasanth, V., Venkatachalapathy, K., Thamarai, L., Parthiban, L., \& Ezhilarasi, T. P. (2017). A survey on cache route schemes to improve QoS in AD-HOC networks. Pakistan Journal of Biotechnology, 14, $265-$ 269.

18. Vasanth, A. V., Venkatachalapathy, K., Latchoumi, T. P., Parthiban, L., Sowmia, T., \& OhmPrakash, V. (2018). An Efficient Cache Refreshing Policy to Improve QoS in MANET Through RAMP. In Proceedings of the Second International Conference on Computational Intelligence and Informatics (pp. 369-381). Springer, Singapore. 